\title{
Impact Scenarios in Boron Carbide: A Computational Study
}

\section{Isaac J. Sugden ${ }^{1}$, David F. Plant ${ }^{2}$ and Robert G. Bell, ${ }^{1, *}$}

${ }^{1}$ University College London, Chemistry Department, 20 Gordon Street, London WC1H 0AJ, UK e-mail: r.g.bell@ucl.ac.uk

${ }^{2}$ AWE, Aldermaston, Reading, RG7 4PR, UK

The effect of radiative impacts on the structure of boron carbide has been studied by both classical and ab initio simulations. As a part of this study, a new forcefield was developed for use in studying boron carbide materials. Impact scenarios in boron carbide were simulated in order to investigate the exceptional resistance of this material, and other icosahedral boron solids, to high-energy impact events. It was observed that interstitial defects created by radiative impacts are likely to be quenched locally, utilising the high substitutional disorder of chains and cages in the boron carbide structure, rather than via impacted atoms recombining with their vacated lattice site.

\section{Introduction}

Boron carbide is a solid based on $\mathrm{B}_{12}$ icosahedra connected in hexagonal sheets. These layers are connected via linear chains along the trigonal [001] direction, with one chain per icosahedron. In the general form, the icosahedra are $\mathrm{B}_{12}$ units and the chains are C-B-C units, making three tetrahedral bonds to icosahedra at either end, as seen in Figure 1. This system does not conform to Wade's rules ${ }^{1}$ in that each chain-icosahedron unit has an unpaired electron. As a result the true structure of boron carbide is disordered, with the nature of disorder not fully characterised: carbon composition can vary in the range 9-20 atom $\%^{2,3}$, whilst X-ray experiments do not distinguish between carbon and boron atoms ${ }^{4}$, and neutron diffraction is problematic when analysing boron solids, due to neutron capture events with ${ }^{10} \mathrm{~B}$ nuclei, although enrichment with ${ }^{11} \mathrm{~B}$ has allowed for some characterisation ${ }^{4}$. Theoretically the lowest energy structure ${ }^{5}$ of stoichiometric $\left(\mathrm{C}_{3} \mathrm{~B}_{12}\right)$ boron carbide would contain solely $\mathrm{CB}_{11}$ cages, and $\mathrm{C}-\mathrm{B}-\mathrm{C}$ chains. This represents the most carbon-rich end of the compositional range, whilst the boron-rich end has a high proportion of $\mathrm{B}_{12}$ icosahedra and $\mathrm{B}-[\mathrm{vacancy}]-\mathrm{B}$ chains. However, it is accepted that the composition of any boron carbide system will include some $\mathrm{C}_{2} \mathrm{~B}_{10}, \mathrm{CB}_{11}$, and $\mathrm{B}_{12}$ cages, and some C-B-C, C-B-B, B-B-B, and 2 or 4 atom chains ${ }^{6}$. It may therefore be regarded as a "frustrated" system, without an ideal structure?

Boron carbide is one example of a family of solids based on layers of boron icosahedra with a double tetrahedral X-X or X-B-X chain of hetero atoms. Other examples include boron phosphide ${ }^{8}$, arsenide $^{9}$, and oxide ${ }^{10}$, as well as $\beta$-boron. Boron carbide is the most conspicuous example of this family, with a large body of experimental data available, due to its uses in varying fields; for example, as a component in control rods in nuclear reactors ${ }^{11}$ and body and vehicle armour ${ }^{12}$, taking advantage of the high neutron absorption cross-section of ${ }^{10} \mathrm{~B}$ and the extreme hardness of boron carbide. For this reason, 
in this work we focus solely on the boron carbide material. However, all of the solids exhibit the same exceptional resistance to radiation. Previous studies in which "icosahedral" boron solids are exposed to radiation have involved the bombardment of $\mathrm{B}_{12} \mathrm{P}_{2}$ with $400 \mathrm{keV}$ electrons ${ }^{14}$, and of $\beta$-boron with $160 \mathrm{keV} \mathrm{N}^{+}$ions ${ }^{13,14}$. In both cases the radiative impact energies are five orders of magnitude greater than common bond dissociation energies, but the crystal structure appeared to remain unaltered in both cases. When boron carbide itself is irradiated with thermal neutrons, lithium and helium ions are produced by neutron capture events of ${ }^{10} \mathrm{~B}$, which cause bubbles to form in the structure. This does not affect nano-crystallinity ${ }^{15,16}$, although micropores are observed. Amorphisation is possible using $\mathrm{H}^{+}$ion radiation, which alters the structure chemically ${ }^{17}$, but $1 \mathrm{MeV}$ electron impacts again do not affect the gross crystal structure ${ }^{18} ; 2 \mathrm{MeV}$ impacts are required to induce amorphisation through impact.

This resistance of the material to structural damage has been attributed to two possible mechanisms. One hypothesis is that crystallinity is retained by a "self-healing" method, whereby impacted atoms are displaced as positive ions, leaving behind the electrons occupying the cage bonding orbitals. Over the timeframe of observation, it is proposed that Coulombic forces drive the displaced atom back to its lattice site ${ }^{13,19}$. An alternative mechanism is one in which the displaced atom is incorporated into a nearby chain, whilst the cage is reformed by extracting an adjacent chain atom. In this way twelve-atom cages are retained whilst chain disorder is increased - an effect that would not readily be observed using crystallographic characterisation methods given the disorder already prevalent in boron carbide chains ${ }^{20}$.

In this work, we examine the proposed mechanisms using classical and ab initio computation. Impact events are simulated using molecular dynamics, in which the velocity of a targeted bulk atom is increased in a random direction. The damage sustained by the bulk solid, after the defect stabilises during the classical MD simulations, is analysed using ab initio techniques.

\section{Methodology}

\subsection{Forcefield derivation}

In order to perform molecular dynamics simulations on the boron carbide systems, forcefield terms needed to be derived. These were validated by comparison with the experimental crystal structure, bulk moduli and vibrational spectra of boron carbide. The bulk modulus was obtained using the Reuss definition provided by the program GULP ${ }^{21}$ (derived from elastic constants calculated for the static system). GULP was also used to compare optimised structures with experiment, whilst an alternative derivation of bulk modulus using molecular dynamics, as well as the main molecular dynamics simulations were performed using DL_POLY $4^{22}$. The system was described as follows.

Given the broad compositional range found among boron carbide samples, the structure was represented using an idealised symmetrical system with $\mathrm{CB}_{11}$ cages and $\mathrm{C}-\mathrm{B}-\mathrm{C}$ chains. For 
computational simplicity the cage carbon atom was placed at the chain bonding "equatorial" site (see Figure 1), implying C1 symmetry.

Bonding within and between cages (intra- and inter-icosahedral bonds), between chains and cages (chain-icosahedral bonds), and chain bonds, were represented by Morse potentials. A harmonic angle term was also required for the chain bend. Non-bonding interactions were described by Lennard-Jones potentials. Charges were derived using the Mulliken method from molecular ab initio calculations on the boron carbide system. These were performed using the CP2K code ${ }^{23}$ at the PBE/6-31G* level, and produced the charges given in table 1 . The presence of so many atom types is necessary to differentiate between inter- and intra-icosahedral bonds; a B2-B2 interaction is inter-icosahedral for example, while B2-B* (where B* represents B1, B3, B4 or B5) is intra. However B2, B4 and B5 atoms are considered chemically equivalent. As table 1 shows, the negative charge is localised at the tetrahedral bonding sites, consistent with the higher electronegativity of carbon atoms, and the more directional covalent nature of the bonding in which they participate (in distinction from the aromatic-type bonding of the cages).

Initial parameters where chosen from the OPLS forcefield ${ }^{24}$, and a fitting process was conducted using the least squares fitting algorithm in the GULP program. As previously stated, the properties fitted to were the vibrational spectra, crystal structure and bulk modulus of experimental boron carbide, leading to the forcefield terms given in table 2. The minimum-energy structure, when optimised in GULP at constant pressure, has the cell parameters $a=5.880 \AA, c=12.074 \AA$. Experimentally the lattice constants vary with carbon content and method of preparation. Towards the higher end of carbon content (roughly for $x \geq 2$, where approximate stoichiometry is $\mathrm{B}_{15-\mathrm{x}} \mathrm{C}_{\mathrm{x}}$ ) lattice parameters lie in the ranges $a=5.591-5.653 \AA$ and $c=12.059-12.163 \AA^{4,25,26}$. Our $c$ parameter lies within the reported range, although the $a$ parameter is slightly larger. However it should be borne in mind that ours is a pristine, idealised structure with maximum $C$ content and overall composition $\mathrm{C}_{3} \mathrm{~B}_{12}$. In reality high carbon content is believed to correlate to a high number of boron chain vacancies and other defects. The larger simulated cell volume is thus not inconsistent with the assumptions made in our structural model.

The forcefield also gives a reasonable fit to vibrational spectra ${ }^{27}$ as shown in Figure 2. Experimentally ${ }^{27}$, the peak at $\sim 1600 \mathrm{~cm}^{-1}$ has been attributed to a chain stretch term, $1100 \mathrm{~cm}^{-1}$ is attributed to the inter-icosahedral B-B vibrations, and the peaks at $800-900 \mathrm{~cm}^{-1}$ to intra-icosahedral B$\mathrm{B}$ vibrations, corresponding to the assignments in this investigation. We note that previous authors ${ }^{28}$ maintain that the Raman band at around $1600 \mathrm{~cm}^{-1}$ arises solely from the presence of carbonaceous impurities. However later works (e.g. ${ }^{29}$ ) have not always concurred with this assignment, and moreover it seems at least fairly conclusive that the band appears in the infrared spectrum of the "pure" material ${ }^{27}$, our simulations not distinguishing between infrared, Raman-active or other modes. It is acknowledged that peaks below $\sim 500 \mathrm{~cm}^{-1}$, indicative of whole lattice vibrations, are not well modelled; however classical simulations typically do not model this area of the spectrum well, and moreover there are no negative frequencies. 
The experimental bulk modulus of boron carbide is variously quoted as $236-247 \mathrm{GPa}^{30}$, and the bulk modulus calculated by GULP using this forcefield is $240 \mathrm{GPa}$. The modulus was also calculated using molecular dynamics: an MD run was performed at ambient temperature, on a 60750 atom system (i.e. a $15 \times 15 \times 6$ supercell), at a high pressure (60 MPa), having been first equilibrated at $1 \mathrm{~atm}$. The bulk modulus was then derived using the following equation:

$$
K=-V\left(\frac{d P}{d V}\right)
$$

where $\mathrm{K}, \mathrm{V}$ and $\mathrm{P}$ represent bulk modulus, volume and pressure respectively. This led to a modulus of $247 \mathrm{GPa}$, within the range of experimental values and very close to the value derived by the Reuss method.

\subsection{Methodology: simulations}

Having derived the forcefield and tested it against various experimental properties, impact studies were performed using DL_POLY 4 on a 15 x 15 x 6 supercell of volume around $574000 \AA^{3}$, containing 60750 atoms. All simulations were carried out using the NPT ensemble at $298 \mathrm{~K}$, and 1 atm pressure, using a 0.001 ps timestep and $12.0 \AA$ short-range cutoff. The Berendsen thermostat and barostat were used, with both time constants set to $1.5 \mathrm{~s}$. Bonded atoms whose separation exceeds that of the bonding cut-off (the sum of their covalent radii) during the course of the simulation lose the attribute of being bonded, i.e. atoms which become significantly displaced due to impact continue to interact via their electrostatic and van der Waals parameters.

Initial simulations were carried out to test the suitability of a range of impact energies, i.e. that the energies are sufficient to cause a reasonable number of atomic displacements, but not so great as to create instability in the simulation. These involved increasing the velocity of a randomly selected atom in a random direction by applying impact energies between 0.1 and $1.2 \mathrm{keV}$.

Further elastic impacts were then simulated by increasing the velocity of a targeted atom in a random direction with impact energy of between 0.1 and $1.1 \mathrm{keV}$. 11 simulations were performed, on each of four atom types (B1, B3, C1 and C4) creating a library of "damaged" phases.

Some of damaged systems were further analysed at an $a b$ initio level, extracting the atom positions of the original 45 atom unit cell that includes the impacted atom, as well as 1 unit cell in the positive and negative $\mathrm{z}$ direction, and the positive $x$ and $y$ directions (effectively a $2 \times 2 \times 3$ supercell of the structure surrounding the impacted atom), for a 540 atom cell of approximate volume $5100 \AA^{3}$ from the DL_POLY molecular dynamics runs. This is a compromise between dilution of defect and computational cost. Single point calculations, geometry optimisations, and $a b$ initio molecular dynamics were then carried out using the $\mathrm{CP} 2 \mathrm{~K}$ program at the $\mathrm{PBE} / 6-31 \mathrm{G}^{*}$ level of theory.

Finally, further forcefield-based MD simulations were carried out using a larger impact energy of $12 \mathrm{keV}$. This is still low in comparison to the energies of experimental radiative impacts, but results in 
much larger atom displacements, allowing the effect of an impact to be assessed at greater distances from the original site of the impacted atom.

\section{Results and discussion}

\subsection{Low energy impacts: Forcefield-based MD studies}

Initial calculations were performed by simulating impacts on a random selection of bulk atoms, confirming that $0.1-1.2 \mathrm{keV}$ is an appropriate range of impact energies to apply under these conditions (60750 atom cell, 0.001 ps timestep, $12 \AA$ cutoff), in that a good number of displacement events were observed, but the energies were not so high so as to render the bulk of the simulations unstable. The changes in cell volume from these preliminary studies are illustrated in Figure 3, showing that the initial impact results rapidly in a slight increase in the cell volume up to about $0.12 \%$, dependent on the impact energy. The plots for each energy represent a single MD run. In general, higher energies caused greater volume changes, although the correlation is not exact, suggesting that the identity of the displaced atoms and the direction of the impact vector are also important factors. Over the course of the simulations ( 8 ps), the volume tends to reduce again for the higher-energy impacts but does not return to the original value. However the majority of the simulations were well-behaved in terms of energy conservation and temperature stability. Only those at 1.0 and $1.1 \mathrm{keV}$ failed due to initial instability in the simulation.

Further impact simulations were carried out as described in section 2.2 on the four representative atom types, with the impact energy imparted in a random direction at each of the 11 energies. Displacements of the impacted atoms 2.5 ps after impact are shown in Table 3. At each energy, the data represent a single impact simulation. These data give an indication as to whether a defect has been generated, 2.5 ps being a timespan over which the atomic positions found to have sufficiently stabilised. Dashes in the table indicate instances where the simulations failed due to excessive cell volume changes. Similarly, $12 \AA$ being the short-range cut-off, any atom displacements greater than this distance caused DL_POLY to fail. Below a cut-off of $\sim 0.6 \AA$ maximum displacement, the structure is regarded as having retained its original structure, while above $3.0 \AA$ a vacancy/interstitial defect has been generated, with an atom displaced from its lattice position. In the results given in Table 3, it can be seen that there is a clear distinction between these two types of outcome, with no displacement observed between 0.50 and $3.02 \AA$.

Broadly, below impact energies of $0.8 \mathrm{keV}$, displacements are consistent with crystallographic positions being retained. However there is evidence that some atom types are more easily displaced that others, for instance, B3 (chain boron) at $0.2,0.5$ and $0.7 \mathrm{keV}$ impact (as well as higher energies where displacement exceeded the short-range cutoff), also suggesting that the direction of impact (chosen randomly in our simulations) is important in determining whether an atom will be displaced. At impact energies higher than $0.8 \mathrm{keV}$, combined interstitial/vacancy defects are formed by displacement of the 
$\mathrm{C} 1, \mathrm{~B} 1$ and B3. Stable defect structures were generated for $\mathrm{C} 1$ at $0.9 \mathrm{keV}$ and $\mathrm{B} 1$ at $1.1 \mathrm{keV}$, in addition to the B3 cases already mentioned.

The effect on local structure for the example of a $1.1 \mathrm{keV}$ impact on a B1 (cage) atom is displayed in Figure 4, which had a defect energy of $\sim 2800 \mathrm{~kJ} \mathrm{~mol}^{-1}$, calculated by comparing the average energies of damaged and undamaged structures in the MD simulation. Following the displacement of the B1 atom, it continued to occupy its interstitial position for the remaining $24 \mathrm{ps}$ of the simulation.

Other defect scenarios arising from impact on the B3 (chain) atoms are shown in Figure 5. As can be seen, the chain boron atoms, following impact, find stable defect positions between layers of icosahedra, in the "pocket" formed between three icosahedra in one layer, and one icosahedron in another layer, shown more clearly in Figure 6. In the case of Figure 6(b), the impacted atom is still bonded to one of the chain carbons; in this case the direction of the random impact generated was mainly along the $z$ direction.

These low-energy impact classical simulations are not inconsistent with either of the selfhealing hypotheses, in that, for instance, $6.75 \AA$ is a distance over which Coulombic attraction is significant. Migration of the impacted atom back to its lattice site could thus conceivably be achieved within the period of experimental observation - a timescale however that would be unreasonable to model using $a b$ initio MD. On the other hand, we would not expect to observe incorporation into other chain moieties with classical atomistic MD.

\subsection{High energy impacts: Forcefield-based MD study}

To compare with the low energy impact simulations, the conditions were altered such that an impact of $12 \mathrm{keV}$ on an icosahedral B1 atom could be simulated. The short-range cutoff was extended to $36 \AA$, to permit atoms to become significantly separated without causing the simulation to terminate.

At this higher impact energy (still an order of magnitude lower than experimental energies of $\sim 160 \mathrm{keV}^{13}$ ), the $\mathrm{B} 1$ atom is displaced by $20 \AA$ after $1.0 \mathrm{ps}$. This result thus argues strongly against the "self-healing" mechanism proposed by Emin et. al., since Coulombic interactions between the impacted atom and its original lattice site would be considerably weaker at this separation, especially when it is considered that the vacancy and interstitial sites are shielded by six icosahedral units. In Figure 7 the progressive damage to the system caused by the impact is illustrated. Following the impact on a single atom of $12 \mathrm{keV}$, it can be seen that a significant region of the structure around the B1 atom is disrupted, with those atoms displaced by at least $1.5 \AA$ being highlighted. The size of the disrupted area reaches a maximum at around $0.5 \mathrm{ps}$.

This result is consistent with the idea that damaged icosahedra subtract a chain atom to complete the cage bonding set, whilst the impacted atom exists as an interstitial, or combines with a nearby chain. This is unlikely to affect the observed long-range crystallinity of the system, since there is an accepted random nature to the composition of chains ${ }^{31}$. 


\subsection{Periodic Ab initio study}

In addition to classical forcefield-based simulation, periodic $a b$ initio molecular dynamics calculations were carried out on the damaged system. As described above, defects generated by low energy impacts were analysed by extracting the local structure around the defect. This 540 atom supercell includes the impacted atom and its original cage. For the case of the $1.1 \mathrm{keV}$ impact on a B1 (originally cage) atom, geometry optimisation of the damaged system (after 24 ps classical MD simulation) found a local minimum in which the impacted boron atom inserts itself into a nearby chain, between a chain carbon and a cage boron atom (see Figure 8). A "psuedo 4-atom" C-B-C-B chain is formed in which the geometry is somewhat distorted compared to the undisturbed structure. The ideally linear chain C-B-C angle becomes $161.2^{\circ}$ and the angles around the tetrahedral chain carbons also distort, with $\mathrm{B}_{\text {chain }}-\mathrm{C}_{\text {chain }}-\mathrm{B}_{\text {cage }}$ ranging up to $132.6^{\circ}$ (ideally $114.9^{\circ}$ ). The $\mathrm{B}_{\text {chain }}-\mathrm{C}_{\text {chain }}$ bond lengths diverge from around $1.50 \AA$ to 1.475 and $1.539 \AA$, and the $\mathrm{C}_{\text {chain }}-\mathrm{B}_{\text {cage }}$ bonds are reduced from an average of $1.797 \AA$ to $1.737 \AA$, whilst in the icosahedron bonded to the interstitial $\mathrm{B}$, the average B-B bond length decreases from $\sim 1.89 \AA$ to $\sim 1.82 \AA$. By comparison, in the forcefield simulations the $\mathrm{B}_{\text {chain }}-\mathrm{C}_{\text {chain }}$ bond lengths close to the defect equilibrate to $1.43 \AA$ and $1.53 \AA$, the corresponding $\mathrm{B}_{\text {chain }}-\mathrm{C}_{\text {chain }}-\mathrm{B}_{\text {cage }}$ angles vary between $92^{\circ}$ and $124^{\circ}$ and the $\mathrm{C}_{\text {chain }}-\mathrm{B}_{\text {cage }}$ bonds average $1.735 \AA$; the median B-B distance in the impacted cage is around $1.84 \AA$. The C-B-C chain angle reduces to about $175^{\circ}$, however the threebody constraint still applies in the forcefield simulation, so direct comparison with $a b$ initio results is not appropriate. With these changes in geometry, the structure is able to accommodate the interstitial chain boron. Ab initio molecular dynamics carried out at $298 \mathrm{~K}$ for $500 \mathrm{fs}$ saw no further change in the bond connectivity of the impacted system.

The original icosahedral cage, from which the B1 atom was abstracted, assumes a nido configuration, as shown in Figure 9. A nido boron cluster is stable when it has 2 electrons more than the closo equivalent, so it is unsurprising that, overall, these cage atoms see a net change in charge of $0.79 e$ from the original structure, consistent with experimental findings on molecular carborane derivatives ${ }^{32}$. While this is not the full formal compensating charge, it demonstrates how the loss of an icosahedral vertex can be partly accommodated through changes in the electronic structure. Further $a b$ initio MD simulations at $298 \mathrm{~K}$ for $500 \mathrm{fs}$ did not see any extraction of nearby chain atoms into the impacted cage, though admittedly this a very short timescale compared to periods of experimental observation. After rebonding of the interstitial B into its new chain, the ab initio defect energy was $783.5 \mathrm{~kJ} \mathrm{~mol}^{-1}$. This indicates that quite substantial impact energies are able to be absorbed via this interstitial-chain bonding mechanism, though also raising questions about the concentration of defects that could be sustained within the material. 


\section{Conclusions}

The effect of radiative impacts on the boron carbide system has been modelled at classical and $a b$ initio (periodic DFT) levels of theory. Previously proposed mechanisms by which the material is able to tolerate the energies of radiative impact without significant structural degradation or amorphisation include (1) self-healing involving recombination of an impacted cage atom with its original cage as a result of electrostatic attraction and (2) insertion of the abstracted atom into a chain unit followed by reformation of the icosahedral cage by a chain atom. Our MD simulations indicate that relatively low impact energies can give rise to significant displacements, e.g. of the order of $20 \AA$ for a $12 \mathrm{keV}$ impact on a cage B atom. Although DFT calculations indeed confirm that negative charge remains on the impacted icosahedral cage, the distances involved are likely to be too large for the self-healing mechanism to be plausible at realistic impact energies. Regarding the second mechanism, a combination of classical and ab initio calculations show that an interstitial boron atom, displaced into a intercage cavity, can then insert into a nearby chain without additional energy barrier. The defect is stabilised both by its distance from the original lattice site and by the extent to which the structure is able to distort to accommodate the additional atom. The original cage remained in a nido-type configuration, stabilised by the additional negative charge. Within the short timescale of our $a b$ initio MD simulations there was no sign of an atom being abstracted from a nearby chain to complete the 12-membered cage. Our simulations therefore provide strong corroboration that the second mechanism is both feasible and likely to explain the origin of the radiation tolerance exhibited by icosahedral boron-containing materials.

However, it is important to consider other experimental evidence regarding the mechanism behind the extraordinary resistance to radiation of icosahedral-boron solids; alongside boron carbide there are several examples of materials that contain icosahedral boron structures, with other atoms occupying chain positions, such as boron phosphide ${ }^{8}$, arsenide ${ }^{9}$ and oxide ${ }^{10}$. There are also several examples of heterosubstituted icosahedra with elements as varied as technetium ${ }^{33}$, tin or germanium at the icosahedral vertices ${ }^{34}$. It is feasible then, that the stabilisation of damaged icosahedra by the abstraction of a chain atom of a different element would be a viable mechanism to resistance of these solids to radiation. Further studies on the stability of such solids are necessary for confirmation.

It is also important to consider that experimentally the radiative bombardment is sufficient to displace each atom several times. Therefore, the combination of an "abstracted chain" and "interstitial boron" defects at a local level will be a route to maintaining crystallinity in these solids, owing to the long term stability of icosahedral units with a vertex removed as a cation, particularly with the degraded cage further stabilised by the partial abstraction of a chain atom.

If possible, more extensive periodic DFT calculations in future could probe the concentration limit of the interstitial chain defects as well as examining the energy barriers to cage recombination and the mechanisms by which the charge imbalances are locally compensated. 


\section{Acknowledgements}

We thank AWE and EPSRC for financial support.

\section{References}

1. Mingos DMP, Polyhedral Skeletal Electron Pair Approach. Acc Chem Res 17: 311-319, 1984.

2. Bouchacourt M, Thevenot F, Melting of Boron-Carbide and the Homogeneity Range of the BoronCarbide Phase. J Less-Common Met 67: 327-331, 1979.

3. Beauvy M, Stoichiometric Limits of Carbon-Rich Boron-Carbide Phases. J Less-Common Met 90 : 169-175, 1983.

4. Morosin B, Kwei GH, Lawson AC, Aselage TL, Emin D, Neutron Powder Diffraction Refinement of Boron Carbides - Nature of Intericosahedral Chains. J Alloys Compd 226: 121-125, 1995.

5. Aydin S, Simsek M, Hypothetically superhard boron carbide structures with a $\mathrm{B}_{11} \mathrm{C}$ icosahedron and three-atom chain. Phys Status Solidi B 246: 62-70, 2009.

6. Domnich V, Reynaud S, Haber RA, Chhowalla M, Boron Carbide: Structure, Properties, and Stability under Stress. J Am Ceram Soc 94: 3605-3628, 2011.

7. Ogitsu T, Gygi F, Reed J, Motome Y, Schwegler E, Galli G, Imperfect Crystal and Unusual Semiconductor: Boron, a Frustrated Element. J Am Chem Soc 131: 1903-1909, 2009.

8. Liu ZL, Kawamura J, Nagasono M, Maeda K, Kawai J, Determination of the structure of boron subphosphide by P Kb X-ray fluorescence spectra. J Electron Spectrosc Relat Phenom 135: 7381, 2004.

9. Ownby PD, Ordered Boron Arsenide. J Am Ceram Soc 58: 359-360, 1975.

10. Kobayashi M, Higashi I, Brodhag C, Thevenot F, Structure of $\mathrm{B}_{6} \mathrm{O}$ Boron-Suboxide by Rietveld Refinement. J Mater Sci 28: 2129-2134, 1993.

11. Inoue $\mathrm{T}$, Onchi $\mathrm{T}$, Koyama H, Suzuki H, Irradiation Effects of Boron-Carbide Used as Control Rod Elements in Fast Breeder-Reactors. J Nucl Mater 74: 114-122, 1978.

12. Jimbou R, Kodama K, Saidoh M, Suzuki Y, Nakagawa M, Morita K, Tsuchiya B, Thermal conductivity and retention characteristics of composites made of boron carbide and carbon fibers with extremely high thermal conductivity for first wall armour. J Nucl Mater 241: 1175-1179, 1997.

13. Emin D, Unusual properties of icosahedral boron-rich solids. J Solid State Chem 179: 2791-2798, 2006.

14. Schmirgeld L, Zuppiroli L, Brunel M, Delafon J, Templier C, Ion Implantations in Boron Remarkable Stability of Covalent Structures Based on Icosahedra. AIP Conf Proc 231: 630-638, 1991.

15. Tarasikov VP, Electron-microscopic investigation of boron carbide irradiated in a fast reactor. Atom Energ 106: 220-224, 2009.

16. Shcherbak VI, Tarasikov VP, Bykov VN, Rudenko VA, Radiation-Damage in Neutron-Irradiated Boron-Carbide. Sov Atom Energy 60: 227-230, 1986.

17. Kushita KN, Hojou K, Furuno S, In-Situ Eels and Tem Observation of Boron-Carbide $\left(\mathrm{B}_{4} \mathrm{C}\right)$ during Hydrogen-Ion and Helium-Ion Bombardments. Microscopy Microanalysis Microstructures 6: 149-157, 1995.

18. Inui $\mathrm{H}$, Mori $\mathrm{H}$, Fujita $\mathrm{H}$, Electron-Irradiation Induced Crystalline to Amorphous Transition in Boron-Carbide. Scripta Metallurgica 22: 249-254, 1988.

19. Carrard M, Emin D, Zuppiroli L, Defect Clustering and Self-Healing of Electron-Irradiated BoronRich Solids. Phys Rev B 51: 11270-11274, 1995.

20. Simeone D, Mallet C, Dubuisson P, Baldinozzi G, Gervais C, Maquet J, Study of boron carbide evolution under neutron irradiation by Raman spectroscopy. J Nucl Mater 277: 1-10, 2000.

21. Gale JD, Rohl AL, The General Utility Lattice Program (GULP). Mol Simul 29: 291-341, 2003.

22. Todorov IT, Smith W, Trachenko K, Dove MT, DL_POLY_3: new dimensions in molecular dynamics simulations via massive parallelism. J Mater Chem 16: 1911-1918, 2006. 
23. Laino T, Mohamed F, Laio A, Parrinello M, An efficient linear-scaling electrostatic coupling for treating periodic boundary conditions in QM/MM simulations. J Chem Theory Comput 2: 13701378, 2006.

24. Jorgensen WL, Tiradorives J, The Opls Potential Functions for Proteins - Energy Minimizations for Crystals of Cyclic-Peptides and Crambin. J Am Chem Soc 110: 1657-1666, 1988.

25. Kwei GH, Morosin B, Structures of the boron-rich boron carbides from neutron powder diffraction: Implications for the nature of the inter-icosahedral chains. J Phys Chem 100: 80318039, 1996.

26. Sologub $\mathrm{O}$, Michiue $\mathrm{Y}$, Mori $\mathrm{T}$, Boron carbide, $\mathrm{B}_{13-\mathrm{x}} \mathrm{C}_{2-\mathrm{y}}(\mathrm{x}=0.12, \mathrm{y}=0.01)$. Acta Crystallogr Sect E: Struct Rep Online 68: i67-i67, 2012.

27. Werheit H, Kuhlmann U, Rotter HW, Shalamberidze SO, Isotopic effects on the phonon modes in boron carbide. J Phys: Condens Matter 22: 2010.

28. Shelnutt JA, Morosin B, Emin D, Mullendore A, Slack G, Wood C, Raman spectroscopy of boron carbides and related boron-containing materials. AIP Conf Proc 140: 312-324, 1986.

29. Shirai K, Emura S, Lattice vibrations and the bonding nature of boron carbide. J Phys: Condens Matter 8: 10919-10929, 1996.

30. Dodd SP, Saunders GA, James B, Temperature and pressure dependences of the elastic properties of ceramic boron carbide (B $\left.\mathrm{B}_{4} \mathrm{C}\right)$. J Mater Sci 37: 2731-2736, 2002.

31. Aselage TL, Tallant DR, Association of broad icosahedral Raman bands with substitutional disorder in $\mathrm{SiB}_{3}$ and boron carbide. Phys Rev B 57: 2675-2678, 1998.

32. Hawthorne MF, Young DC, Wegner PA, Carbametallic Boron Hydride Derivatives .I. Apparent Analogs of Ferrocene and Ferricinium Ion. J Am Chem Soc 87: 1818-1819, 1965.

33. Louie AS, Harrington LE, Valliant JF, The preparation and characterization of functionalized carboranes and Re/Tc-metallocarboranes as platforms for developing molecular imaging probes: Structural and cage isomerism studies. Inorg Chim Acta 389: 159-167, 2012.

34. Gadt T, Dimmer JA, Fleischhauer S, Frank A, Nickl C, Wutz T, Eichele K, Wesemann L, Oxidation of germa- and stanna-closo-dodecaborate. Dalton Trans 44: 4726-4731, 2015. 
Table 1. Atom types and their charges in boron carbide (see fig. 1 for key).

\begin{tabular}{ll}
\hline Atom type & Charge $/ q_{e}$ \\
\hline C1 & -0.896 \\
B3 & 0.7 \\
C3 & -0.68 \\
C4 & -0.576 \\
B1 & 0.1974 \\
B2 & 0.0775 \\
B4 & 0.0775 \\
B5 & 0.0775 \\
\hline
\end{tabular}

Table 2. Forcefield terms used in simulations of Boron Carbide

Bonding terms (Morse potential) $\quad E=D_{0}\left(1-e^{-\alpha\left(r-r_{0}\right)}\right.$

\begin{tabular}{l|lll}
\hline Type & $D_{0} / \mathrm{kJ} / \mathrm{mol}^{-1}$ & $\alpha / \AA^{-1}$ & $r_{0} / \AA$ \\
\hline $\mathrm{B}_{\text {cage }}-\mathrm{B}_{\text {cage }}$ (intra-icosahedral term) & 144.73 & 1.9 & 1.8 \\
$\mathrm{~B}_{\text {cage }}-\mathrm{B}_{\text {cage }}$ (inter-icosahedral term) & 168.85 & 1.9 & 1.715 \\
$\mathrm{~B}_{\text {cage }}-\mathrm{C}_{\text {cage }}$ & 139.90 & 1.9 & 1.760 \\
$\mathrm{~B}_{\text {chain }}-\mathrm{C}_{\text {chain }}$ & 144.73 & 1.9 & 1.433 \\
$\mathrm{C}_{\text {cage }}-\mathrm{C}_{\text {chain }}$ & 144.73 & 1.9 & 1.605 \\
$\mathrm{~B}_{\text {cage }}-\mathrm{Cc}_{\text {hain }}$ & 144.73 & 1.9 & 1.605 \\
\hline
\end{tabular}

Angle terms (harmonic) $\quad E=K\left(\theta-\theta_{0}\right)^{2}$

\begin{tabular}{l|ll}
\hline Type & $K / \mathrm{kJ} \mathrm{mol}^{-1}$ & $\theta_{0} /{ }^{\circ}$ \\
\hline C-B-C & 482.4152 & 180.0 \\
\hline
\end{tabular}

Non-bonding terms (Lennard-Jones potential) $E=4 \varepsilon\left[\left(\frac{\sigma}{r}\right)^{12}-\left(\frac{\sigma}{r}\right)^{6}\right]$

\begin{tabular}{ccc}
\hline Type & $\varepsilon / \mathrm{kJ} \mathrm{mol}^{-1}$ & $\sigma / \AA$ \\
\hline B-B & 0.397132 & 3.453000 \\
B-C & 0.317752 & 3.411000 \\
C-C & 0.254238 & 2.860000 \\
\hline
\end{tabular}


Table 3. Atom displacements at $2.5 \mathrm{ps}$ following impact in classical MD simulations

\begin{tabular}{c|cccc}
\hline $\begin{array}{c}\text { Impact } \\
\text { Energy } \\
/ \mathrm{keV}\end{array}$ & $\mathrm{C} 4$ & $\mathrm{C} 1$ & $\mathrm{~B} 1$ & $\mathrm{~B} 3$ \\
\hline 0.1 & 0.08 & 0.44 & 0.08 & 0.34 \\
0.2 & 0.16 & 0.24 & 0.26 & 4.05 \\
0.3 & 0.32 & 0.38 & 0.18 & 0.20 \\
0.4 & 0.20 & 0.28 & 0.09 & 0.05 \\
0.5 & - & 0.50 & 0.07 & 3.02 \\
0.6 & 0.30 & $>12$ & 0.14 & 0.34 \\
0.7 & 0.23 & - & 0.19 & 3.59 \\
0.8 & - & $>12$ & 0.16 & - \\
0.9 & - & 9.38 & 0.12 & $>12$ \\
1.0 & - & 0.41 & 0.08 & $>12$ \\
1.1 & - & $>12$ & 6.75 & $>12$ \\
\hline
\end{tabular}




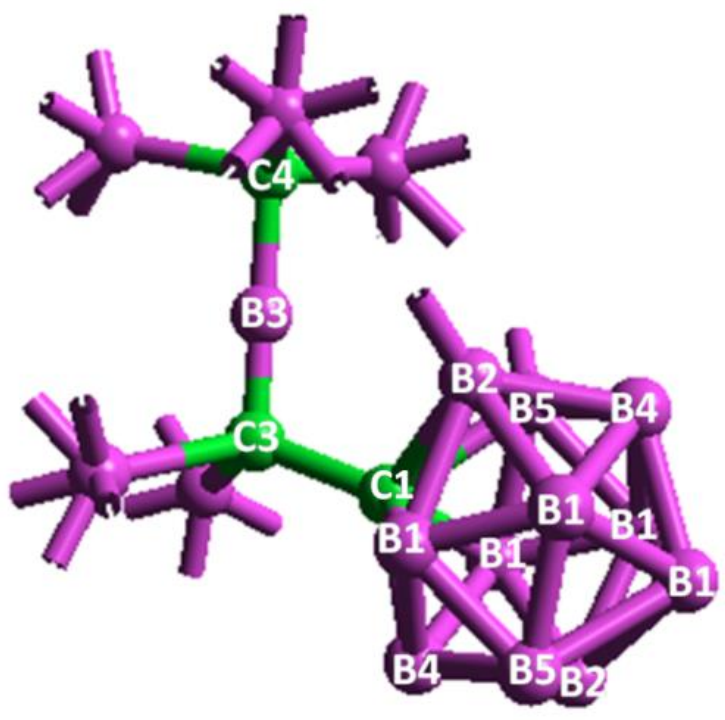

Figure 1. Motif of $\mathrm{C}_{3} \mathrm{~B}_{12}$ with atom types labelled 


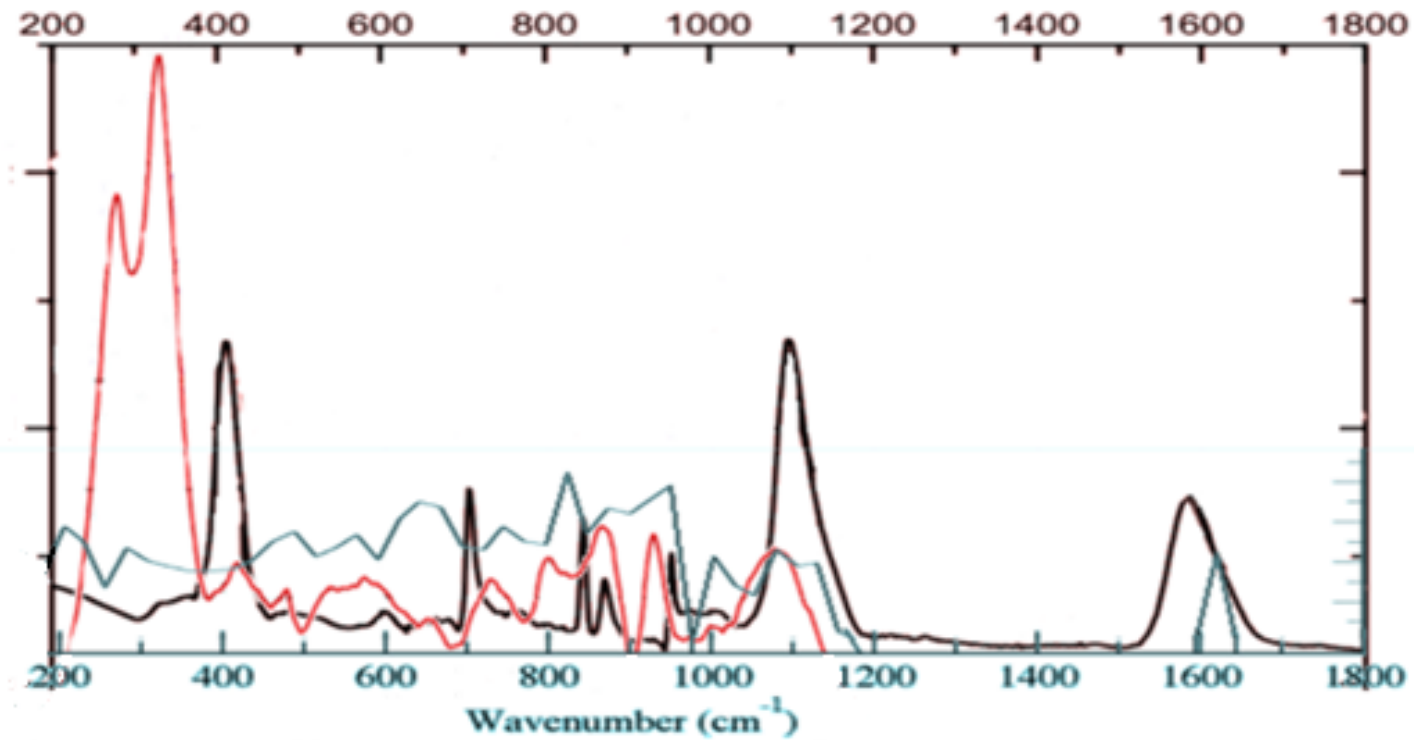

Figure 2. Experimental ${ }^{27}$ infrared (red), Raman (black) and theoretical (blue) vibrational spectra of boron carbide 


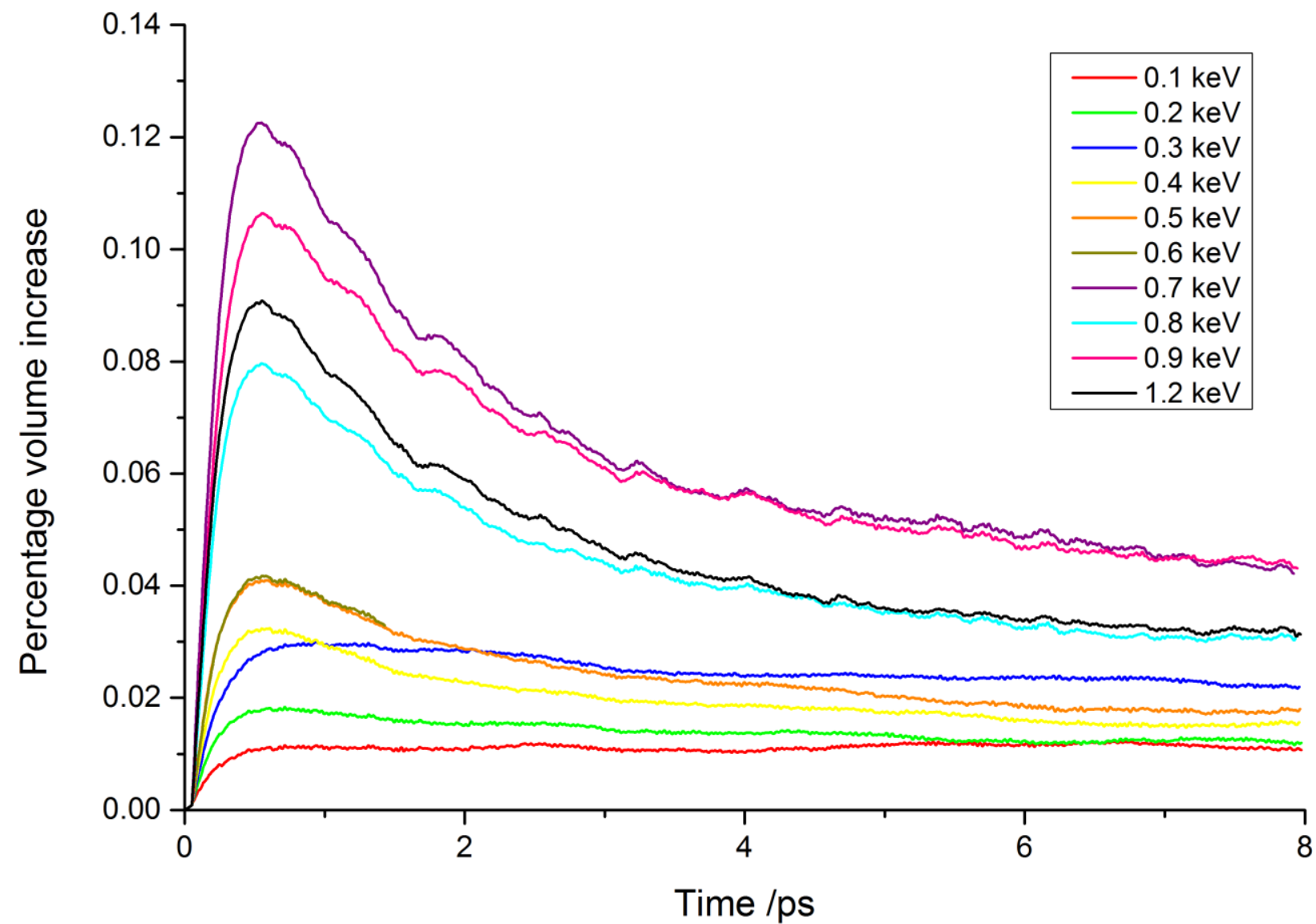

Figure 3. Evolution of cell volume following impacts at different energies. Impacted atom and direction of velocity were chosen at random in each case. 

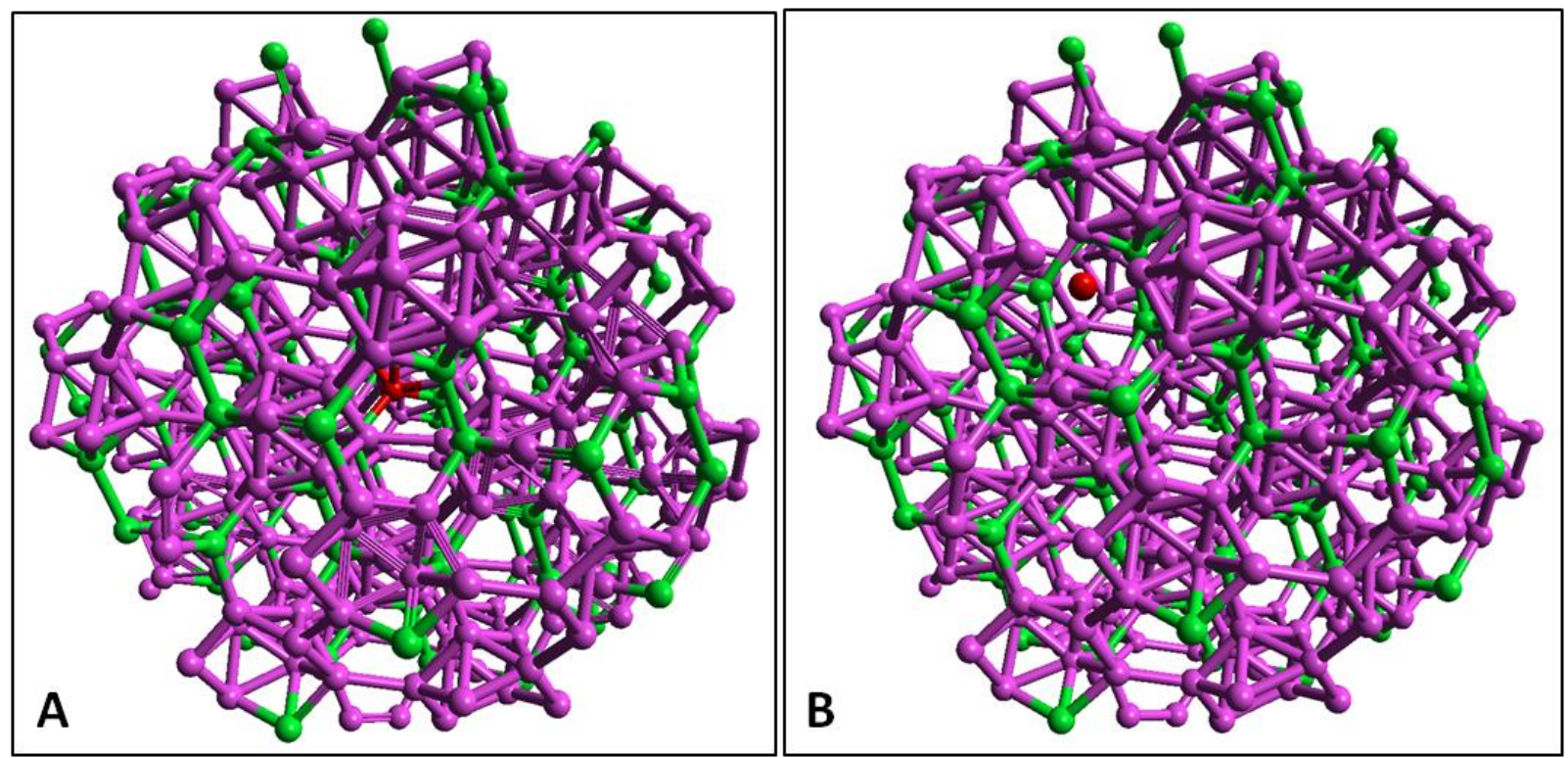

Figure 4. Fragment of Boron Carbide structure within $10 \AA$ radius of impacted B1 atom, shown in red before (A) and after (B) impact.. 


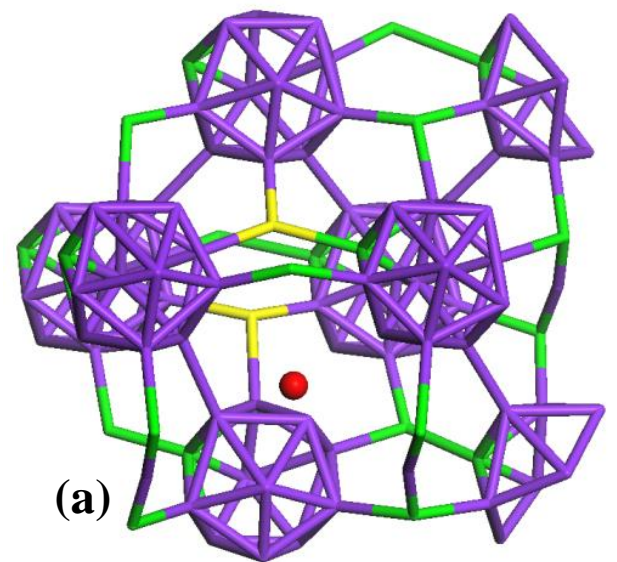

(b)

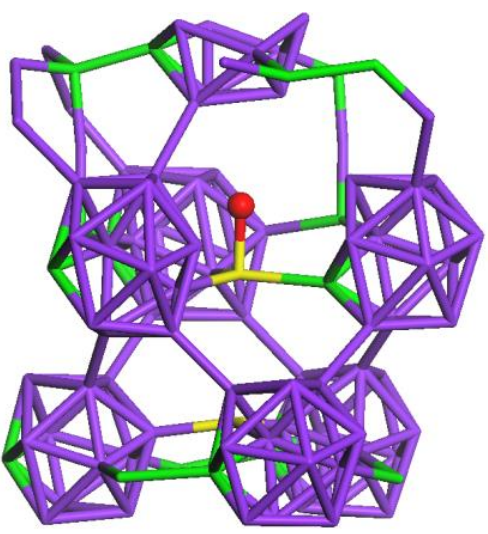

(c)

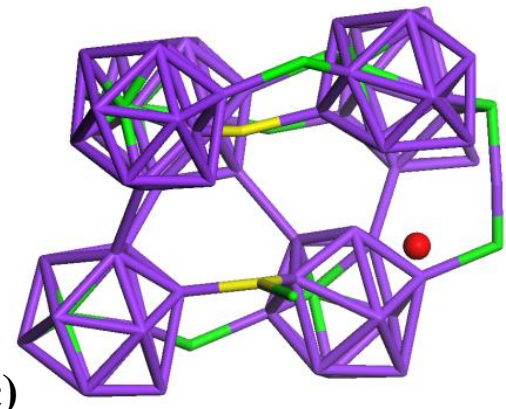

Figure 5. Fragments of boron carbide structure showing B3 displacement during classical MD simulation, following impacts of (a) $0.2 \mathrm{keV}$, (b) $0.5 \mathrm{keV}$, (c) $0.7 \mathrm{k} \mathrm{eV}$. Impacted atoms are shown as red spheres, whilst the chain atoms with which they were initially bonded are shown in in yellow (otherwise green: C; purple: B) 


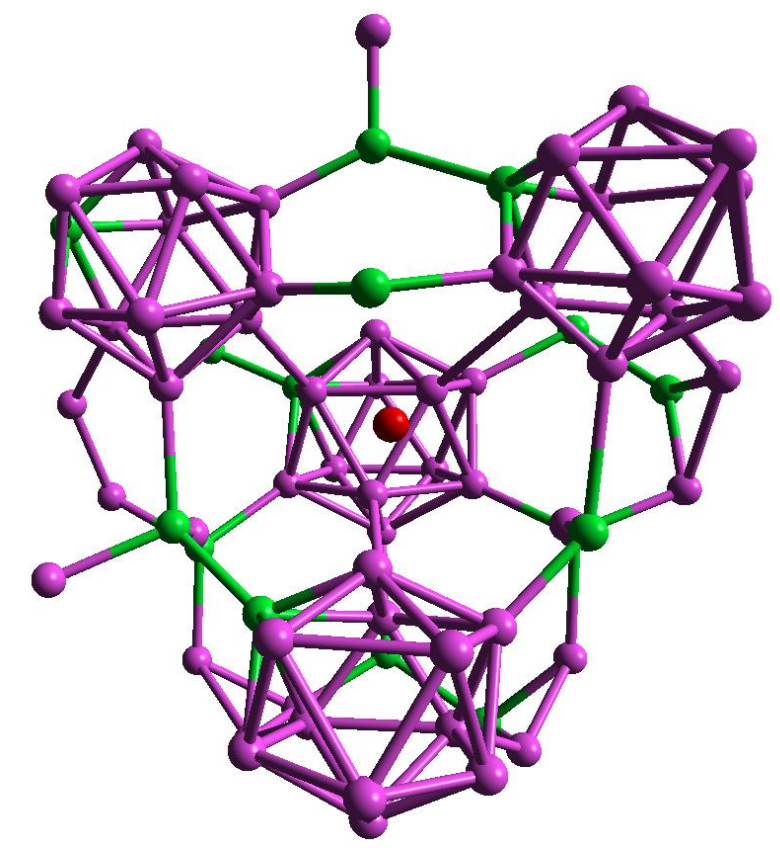

Figure 6. View of boron carbide structure showing interstitial position between four icosahedra (interstitial atom highlighted in red). 


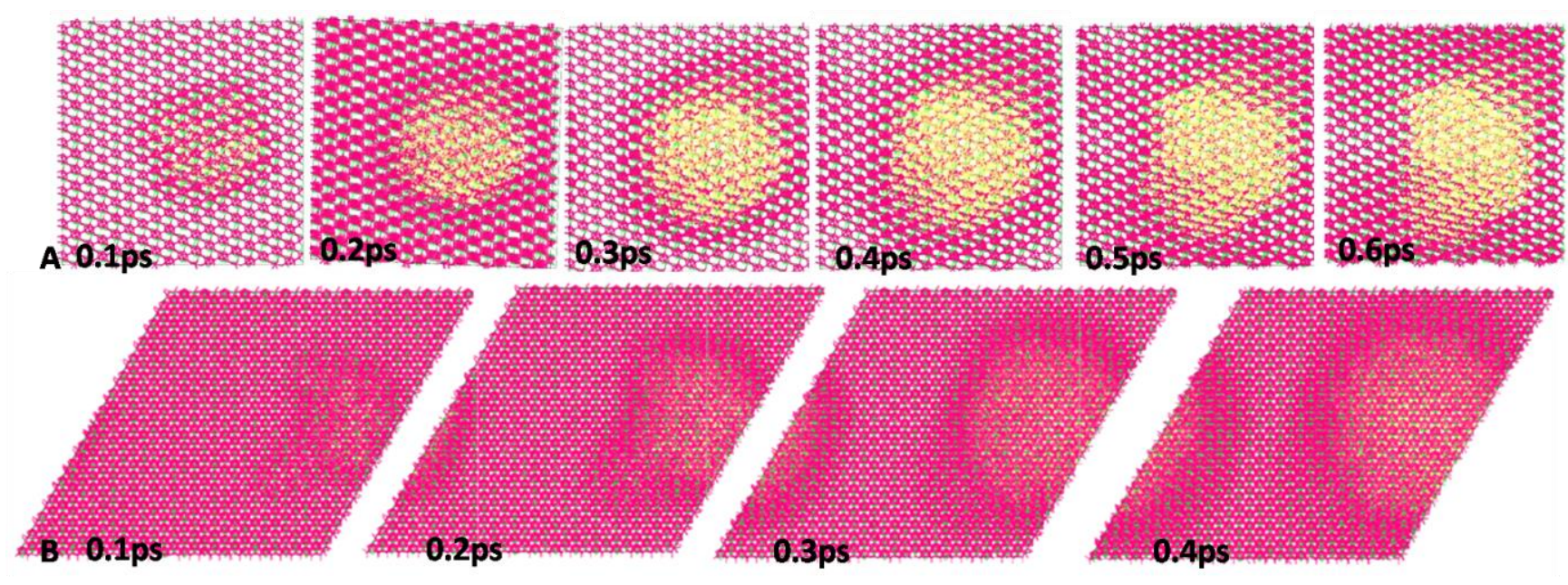

Figure 7. Simulation cell of Boron Carbide (60750 atoms), at $0.1 \mathrm{ps}$ intervals following $12 \mathrm{keV}$ impact on a B1 atom, viewed (A) along the axis of impact and (B) perpendicular to impact.. Atoms that are displaced by more than $1.5 \AA$ are highlighted in yellow. 


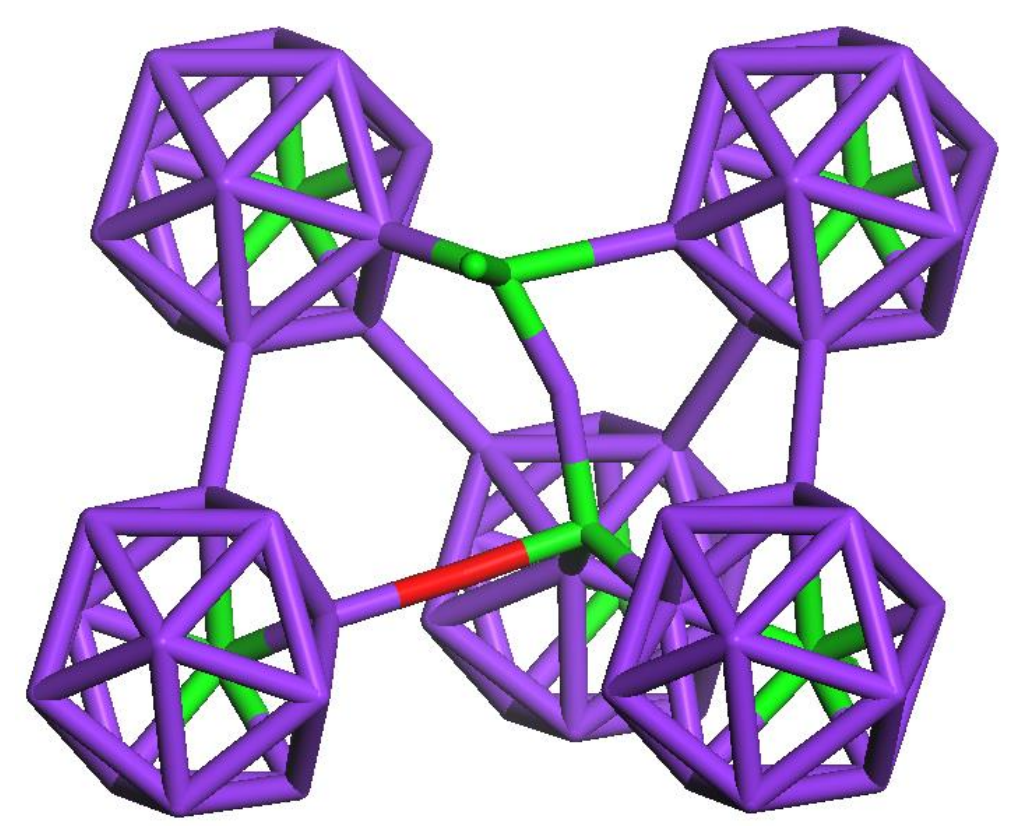

Figure 8. Fragment of the boron carbide structure showing the insertion of impacted boron atom into chain. Colour code - red: original impacted B atom; purple: other B atoms; green - C atoms. 


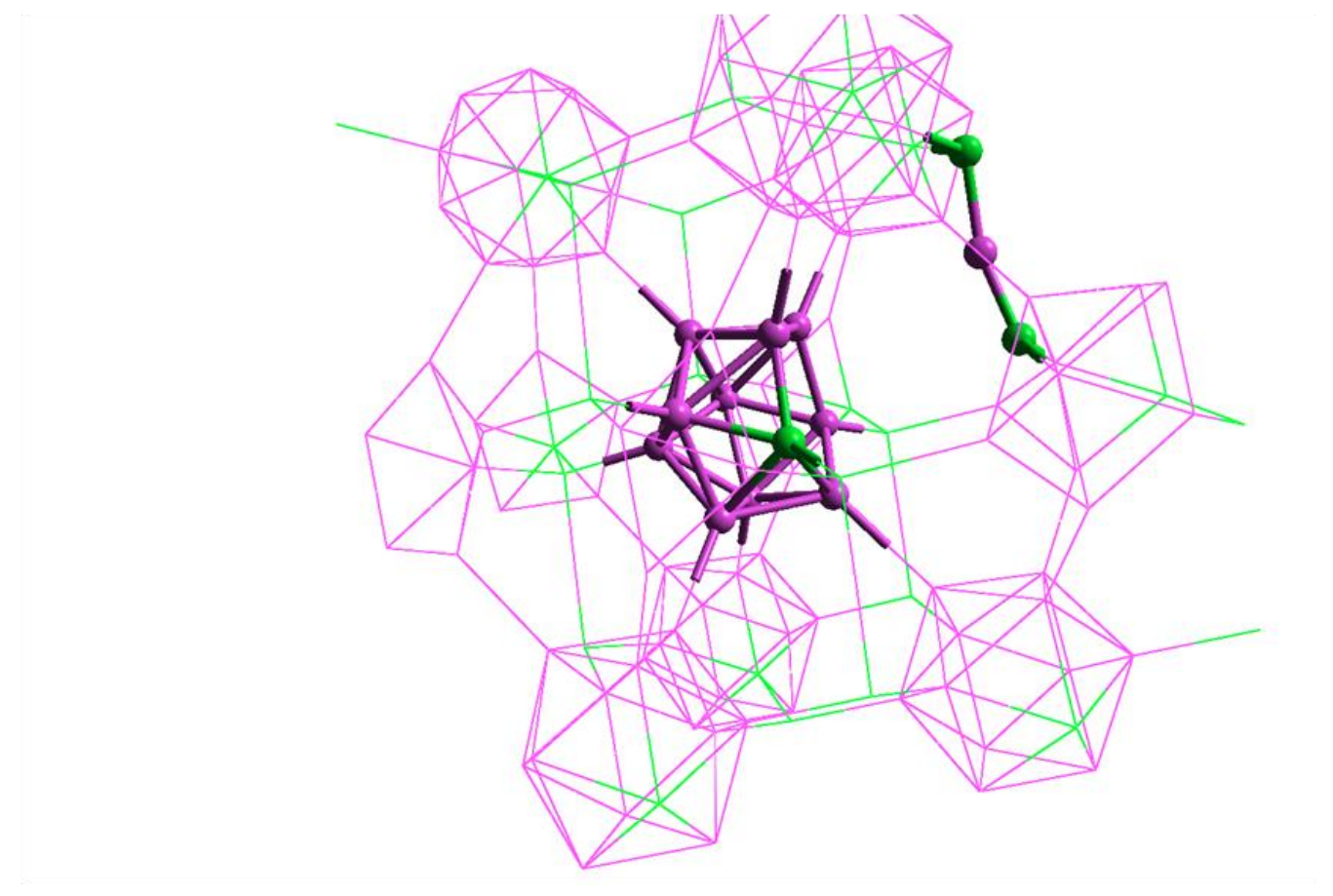

Figure 9. Fragment of impacted boron carbide structure showing residual cage with nido configuration, highlighted with spheres. The closest C-B-C chain is also highlighted. 